\title{
New Developments in Treacherous Points of Light-Front Dynamics
}

\author{
Chueng-Ryong $\mathrm{Ji}^{\mathrm{a} *}$, Bernard L. G. Bakker ${ }^{\mathrm{b}}$ and Ho-Meoyng Choi ${ }^{\dagger}{ }^{\dagger}$ \\ ${ }^{a}$ Department of Physics, North Carolina State University, Raleigh, NC 27695-8202, USA \\ ${ }^{\mathrm{b}}$ Department of Physics and Astronomy, Vrije Universiteit, Amsterdam, The \\ Netherlands \\ ${ }^{\mathrm{c}}$ Department of Physics, Teachers College, Kyungpook National University, Daegu, \\ Korea 702-701
}

Light-front dynamics(LFD) plays an important role in hadron phenomenology. The last few years, however, it has been emphasized that treacherous points such as zeromode contributions should be taken into account for successful LFD applications to hadron phenomenology. We discuss some examples of treacherous points and present new progress made the last few years to handle them correctly.

\section{INTRODUCTION}

Light-front dynamics (LFD) provides a unified framework to analyze various experimental measurements such as generalized parton distributions (GPDs) and single spin asymmetries (SSA) at JLab and DESY (Hermes) [ 1], B-decays at SLAC (BaBar) and KEK (Belle) [2] as well as quark gluon plasma (QGP) production at BNL (RHIC) and CERN (ALICE) [ 3], etc.. Owing to the rational energy-momentum dispersion relation, LFD has distinct features compared to other forms of Hamiltonian dynamics. In particular, the vacuum fluctuations are suppressed and the kinematic generators are proliferated in LFD. Overall, these distinct features can be regarded as advantageous in hadron phenomenology. However, LFD implies also treacherous points as one may realize from the significance of zero-mode contributions even in the good $(+)$ current analyses [ 4]. It has also been shown that the common belief of equivalence between manifestly covariant calculations and naive light-front (LF) calculations is not always realized [5] unless treacherous points are well taken care of [ 6]. Thus, careful investigations of treacherous points and judicious ways of handling them should be imperative for LFD to be distinctively useful compared to other forms of Hamiltonian dynamics [ 7 .

In this presentation, we discuss an example that requires the inclusion of the arc contribution in the light-front energy contour integration in order to achieve equivalence between the LFD result and the manifestly covariant result. For a concrete example of a zero-mode contribution, we also summarize the Standard Model analysis of the vector anomaly in the CP-even form factors of $W^{ \pm}$gauge bosons. We then present a power

\footnotetext{
*Supported by Department of Energy under the contract DE-FG02-96ER40947.

${ }^{\dagger}$ Supported by Korean Research Foundation under the contract KRF-2005-070-C00039.
} 
counting method to correctly pin down which hadron form factors receive a zero-mode contribution and which ones do not.

\section{ARC CONTRIBUTION}

It is well known that spurious divergences appear in LF calculations that do not appear when the same amplitudes are computed in the standard equal-time formulation. This has been taken to mean that the equivalence between the LF and manifestly covariant formalisms is not complete. These divergences have been regulated with various methods including the principal-value prescription, the Mandelstam-Leibbrandt prescription, cutoffs, smearing, and BPHZ-like differentiation and reintegration. We encountered an example of such a divergence in one of our previous works [5]. There, an end-point singularity was seen in the (1+1)-dimensional calculation of the pseudoscalar (and scalar) elastic form factor. Recently, we have shown [6] that the integrand in this case does not vanish sufficiently fast as the LF energy $\left(k^{-}\right)$goes to infinity and the arc contribution is nonzero so that it must be included in order to obtain the correct result. We also showed [ 6] that point contributions occur when the integration contour in the $k^{-}$plane crosses a moving singularity.

The key point of the arc contribution may be seen from the following matrix element:

$$
<p^{\prime}\left|J^{-}\right| p>=2 N \int \frac{d k^{+} d k^{-}}{(2 \pi)^{2}} \frac{\left(m^{2}-k^{+} k^{-}\right)\left(k^{-}-p^{-}-p^{\prime-}\right)-k^{+} p^{-} p^{-}}{k^{+}\left(k^{+}-p^{+}\right)\left(k^{+}-p^{\prime+}\right)\left(k^{-}-k_{1}^{-}\right)\left(k^{-}-k_{2}^{-}\right)\left(k^{-}-k_{3}^{-}\right)},
$$

where $k_{1}^{-}=\frac{m^{2}-i \epsilon}{k^{+}}, k_{2}^{-}=p^{-}+\frac{m^{2}-i \epsilon}{k^{+}-p^{+}}, k_{2}^{-}=p^{-}+\frac{m^{2}-i \epsilon}{k^{+}-p^{+}}$. We see from this equation that the numerator has a term proportional to $\left(k^{-}\right)^{2}$ while the highest power of $k^{-}$in the denominator is 3 . The numerator has also $d k^{-}$, so, if we set $k^{-}=R \exp i \theta$ to compute the contribution from the arc of the $k^{-}$contour integration, then we find that the term proportional to $\left(k^{-}\right)^{2}$ in the numerator has no $1 / R$ suppression factor and can contribute to the form factor calculation.

For the $k^{+}<0$ region, all three poles $k_{1}^{-}, k_{2}^{-}, k_{3}^{-}$are in the upper half-plane and thus by closing the contour in the lower half-plane we get

$$
\int_{-\infty}^{+\infty} d k^{-}=-\int_{\operatorname{arc}} d k^{-}
$$

From this, we find the following contribution for the $k^{+}<0$ region:

$$
<p^{\prime}\left|J^{-}\right| p>=\frac{2 N}{(2 \pi)^{2}} \int_{-\infty}^{0} d k^{+} \frac{-i \pi}{\left(k^{+}-p^{+}\right)\left(k^{+}-p^{+}\right)} .
$$

Similary, we find the arc-contributions from the other regions; $0<k^{+}<p^{+}, p^{+}<k^{+}<$ $p^{\prime+}$, and $k^{+}>p^{+}$. Summing all arc-contributions for the entire $k^{+}$region, we get

$$
\begin{aligned}
<p^{\prime}\left|J^{-}\right| p> & =\frac{i 2 N \pi}{(2 \pi)^{2}}\left[-\int_{-\infty}^{0}-\int_{0}^{p^{+}}+\int_{p^{+}}^{p^{+}}+\int_{p^{+}}^{\infty}\right] \frac{d k^{+}}{\left(k^{+}-p^{+}\right)\left(k^{+}-p^{++}\right)} \\
& =\frac{i N}{2 \pi}\left[-\int_{-\infty}^{p^{+}}+\int_{p^{+}}^{p^{+}}+\int_{p^{\prime}}^{\infty}\right] \frac{d k^{+}}{\left(k^{+}-p^{+}\right)\left(k^{+}-p^{++}\right)}
\end{aligned}
$$


Here, we can show that the contributions from $\int_{-\infty}^{p^{+}}$and $\int_{p^{\prime+}}^{\infty}$ cancel each other exactly. Thus, we find the following arc-contribution:

$$
<p^{\prime}\left|J^{-}\right| p>=\frac{i N}{2 \pi} \int_{p^{+}}^{p^{+}} \frac{d k^{+}}{\left(k^{+}-p^{+}\right)\left(k^{+}-p^{+}\right)} \text {. }
$$

This contribution leads to the correct result for the physical form factor removing the discrepancy between the $J^{+}$and $J^{-}$calculations.

\section{VECTOR ANOMALY}

Anomalies betray the true quantal character of a quantized field theory. A brief historical remark on the anomaly associated with the fermion-triangle loop was made in Ref. [ 8. and our calculation of the vector anomaly has been presented in detail for the Standard Model (SM) in Refs. [ 9, 10]. In conjunction with the LF singularities [11] presented by Ben Bakker in this conference, we briefly summarize the vector anomaly results including the zero-mode contribution in LFD.

The Lorentz-covariant and gauge-invariant CP-even electromagnetic $\gamma W^{+} W^{-}$vertex is defined [12, 13] by

$\Gamma_{\alpha \beta}^{\mu}=i e\left\{A\left[\left(p+p^{\prime}\right)^{\mu} g_{\alpha \beta}+2\left(g_{\alpha}^{\mu} q_{\beta}-g_{\beta}^{\mu} q_{\alpha}\right)\right]+(\Delta \kappa)\left(g_{\alpha}^{\mu} q_{\beta}-g_{\beta}^{\mu} q_{\alpha}\right)+\frac{\Delta Q}{2 M_{W}^{2}}\left(p+p^{\prime}\right)^{\mu} q_{\alpha} q_{\beta}\right\}$,

where $p\left(p^{\prime}\right)$ is the initial(final) four-momentum of the $W$ gauge boson and $q=p^{\prime}-p$. Here, $\Delta \kappa$ and $\Delta Q$ are the anomalous magnetic and quadrupole moments, respectively. Beyond the tree level,

$A=F_{1}\left(Q^{2}\right), \quad-\Delta \kappa=F_{2}\left(Q^{2}\right)+2 F_{1}\left(Q^{2}\right), \quad-\Delta Q=F_{3}\left(Q^{2}\right)$,

where $F_{1}, F_{2}$ and $F_{3}$ are the usual electromagnetic form factors for the spin-1 particles [ 14.

While the anomalous quadrupole moment $\Delta Q$ (or $\left.F_{3}\left(Q^{2}\right)\right)$ is found to be completely independent of the regularization methods as it must be, we find that the anomalous magnetic moment $\Delta \kappa$ (or $\left.F_{2}\left(Q^{2}\right)+2 F_{1}\left(Q^{2}\right)\right)$ differs by some fermion-mass-independent constants depending on the regularization methods [ 9, 10]. Unless the fermion-massindependent differences are completely cancelled, a unique prediction of $\Delta \kappa$ would be impossible. Within the SM, they completely cancel out owing to the anomaly free condition, i.e. the zero-sum of the charge factors $\left(\sum_{f} Q_{f}=0\right)$ in each generation.

In LFD, we compute the form factors using the following helicity amplitudes $G_{h^{\prime} h}^{+}=$ $\epsilon_{h^{\prime}}^{*} \cdot \Gamma^{+} \cdot \epsilon_{h}$ for the initial and final polarization vectors $\epsilon_{h}$ and $\epsilon_{h^{\prime}}^{*}$, respectively, in the $q^{+}=q^{0}+q^{3}=0$ frame,

$G_{++}^{+}=2 p^{+}\left(F_{1}+\eta F_{3}\right), G_{+0}^{+}=p^{+} \sqrt{2 \eta}\left(2 F_{1}+F_{2}+2 \eta F_{3}\right)$,

$G_{+-}^{+}=-2 p^{+} \eta F_{3}, G_{00}^{+}=2 p^{+}\left(F_{1}-2 \eta F_{2}-2 \eta^{2} F_{3}\right)$,

where $\eta=Q^{2} / 4 M_{W}^{2}$. In the $q^{+}=0$ frame, one might expect that the non-valence contribution is absent since the integration range for the non-valence amplitude is shrunk to zero. However, this is not the case as we pointed out in Ref. [4]. Calling the non-zero 
contribution from the non-valence part in the $q^{+}=0$ frame the zero-mode, we find that only the helicity zero-to-zero amplitude $G_{00}^{+}$receives a zero-mode contribution given by

$$
\left(G_{00}^{+}\right)_{\text {z.m. }}=\frac{g^{2} Q_{f} p^{+}}{2 \pi^{3} M_{W}^{2}} \int_{0}^{1} d x \int d^{2} \vec{k}_{\perp} \frac{\vec{k}_{\perp}^{2}+m_{1}^{2}-x(1-x) Q^{2}}{\vec{k}_{\perp}^{2}+m_{1}^{2}+x(1-x) Q^{2}} \neq 0 .
$$

The zero-mode contribution to $G_{00}^{+}$is essential, because the unwelcome divergences from the valence part due to the terms with a power of the transverse momentum such as $\left(k_{\perp}^{2}\right)^{2}$ are precisely cancelled by the same terms with the opposite sign from the zero-mode contribution. One may attempt to remove the zero-mode using Pauli-Villars regularization. However, we find that such an artificial removal of the zero-mode makes the LF calulcation impossible introducing uncontrollable divergences. The details of our calculations were presented in Ref. [9].

The symptom of vector anomaly in LFD also appears as the violation of the rotation symmetry or the angular momentum conservation (i.e. angular condition [ 15]). This appearance is drastically different from the case of the manifestly covariant calculation. However, the anomaly-free condition $\left(\sum_{f} Q_{f}=0\right)$ in the SM again removes the difference and restores the rotation symmetry and the angular momentum conservation in LFD.

\section{POWER COUNTING METHOD}

Jaus [16] proposed a covariant LF approach involving the lightlike four vector $\omega^{\mu}\left(\omega^{2}=\right.$ 0 ) as a variable and developed a way of finding the zero-mode contribution to remove the spurious amplitudes proportional to $\omega^{\mu}$. Our formulation, however, is intrinsically distinguished from this $\omega$-dependent formulation since it involves neither $\omega^{\mu}$ nor any unphysical form factors. Our method of finding the zero-mode contribution is a direct power-counting of the longitudinal momentum fraction in the $q^{+} \rightarrow 0$ limit for the offdiagonal elements in the Fock-state expansion of the current matrix [4, 17, 18, 19]. This power-counting method is straightforward, since the longitudinal momentum fraction is one of the integration variables in the LF matrix elements (i.e. helicity amplitudes) and the behaviors of the longitudinal momentum fraction in the integrand are known from the helicity amplitudes.

For spin-1 electroweak form factors and using the rather simple (manifestly covariant) vertex $\Gamma^{\mu}=\gamma^{\mu}$, both Jaus and we agree on the absence of zero-mode contributions. However, Jaus and we do not agree when $\Gamma^{\mu}$ is extended to the more phenomenologically accessible ones given by

$\Gamma^{\mu}=\gamma^{\mu}-\frac{\left(k+k^{\prime}\right)^{\mu}}{D}$

where $k$ and $k^{\prime}$ are the relative four momenta for the two constituent quarks. Although Jaus's calculation and our calculation used the same denominator $D$ in Eq. (10), they led to different conclusions in the analysis of the zero-mode contribution. Even if $D$ is chosen in such a way as to get the manifestly covariant $\Gamma^{\mu}$, the difference in the conclusions doesn't go away.

For an example of the weak transition form factors between the pseudoscalar and vector mesons, Jaus [16] concluded that the form factor $A_{1}\left(q^{2}\right)$ [or $f\left(q^{2}\right)$ ] receives a zero-mode 
contribution. We do not agree with his result but find that $f\left(q^{2}\right)$ is free from the zeromode contribution if the denominator $D$ in Eq. (10) contains a term proportional to the LF energy $\left(k^{-}\right)^{n}$ with a power $n>0$. The phenomenologically accessible light-front quark model satisfies this condition $n>0$.

As we have shown in detail in Ref. [19], we can determine the existence/nonexistence

of the zero-mode contribution to $f\left(q^{2}\right)$ by counting the powers of the LF energy $k^{-}$in the denominator. For example, if $D=D_{\text {cov }}(k \cdot P) \equiv\left[2 k \cdot P+M_{V}\left(m_{q}+m_{\bar{q}}\right)-i \epsilon\right] / M_{V}$, where $P$ is the four momentum of the vector meson, see Ref. [20], then $D$ contains a term proportional to the LF energy $\left(k^{-}\right)^{n}$ with the power $n=1$. This power-counting shows that the form factor $f\left(q^{2}\right)$ should not receive a zero-mode contribution in the $D_{\text {cov }}(k \cdot P)$ case. We have confirmed that the results found our way coincide with the ones from the manifestly covariant calculation, while Jaus's method of finding zero-mode contributions has a limitation in the choice of $\Gamma^{\mu}$.

\section{REFERENCES}

1. See, e.g., K. Goeke, M.V. Polyakov and M. Vanderhaeghen, Prog. Part. Nucl. Phys. 47 (2001) 401, and references therein.

2. For a review of B-Physics with emphasis on LFD, see C.-R. Ji and H.-M.Choi, Nucl. Phys. Proc. Suppl. 90 (2000) 93.

3. An example of applying LF wavefunctions to RHIC physics can be seen in R.J. Fries, B. Müller, C. Nonaka and S.A. Bass, Phys. Rev. C 68 (2003) 044902; B.Hong, C.-R.Ji and D.-P.Min, Phys. Rev. C 73 (2006) 054901.

4. B.L.G. Bakker, H.-M. Choi and C.-R. Ji, Phys. Rev. D 65 (2002) 116001.

5. B.L.G. Bakker and C.-R. Ji, Phys. Rev. D 62 (2000) 074014.

6. B.L.G. Bakker, M.A. DeWitt, C.-R.Ji, and Y. Mishchenko, Phys. Rev. D 72 (2005) 076005.

7. P.A.M. Dirac, Rev. Mod. Phys. 21 (1949) 392.

8. C.-R. Ji and B.L.G. Bakker, Few-Body Systems 36 (2005) 137.

9. B.L.G. Bakker and C.-R. Ji, Phys. Rev. D 71 (2005) 053005.

10. C.-R. Ji, B.L.G. Bakker and H.-M. Choi, Nucl. Phys. B, in press hep-ph/0510210].

11. B.L.G. Bakker, J.K. Boomsma and C.-R. Ji, in this Proceedings.

12. W.A. Bardeen, R. Gastmans and B. Lautrup, Nucl. Phys. B 46 (1972) 319.

13. G. Couture and J.N. Ng, Z. Phys. C 35 (1987) 65.

14. B.L.G. Bakker and C.-R. Ji, Phys. Rev. D 65 (2002) 073002.

15. C.Carlson and C.-R.Ji, Phys. Rev. D 67 (2002) 116002.

16. W. Jaus, Phys. Rev. D 60 (1999) 054026; Phys. Rev. D 67 (2003) 094010.

17. B.L.G. Bakker, H.-M. Choi and C.-R. Ji, Phys. Rev. D 67 (2003) 113007.

18. H.-M. Choi and C.-R. Ji, Phys. Rev. D 70 (2004) 053015.

19. H.-M. Choi and C.-R. Ji, Phys. Rev. D 72 (2005) 013004.

20. J.P.B.C. de Melo and T. Frederico, Phys. Rev. C 55 (1997) 2043. 